Contributions to Game Theory and Management, XIII, 449-466

\title{
Bibliography of V. V. Mazalov (В.В. Мазалов) Scientific Papers
}

\author{
Anna N. Rettieva ${ }^{1}$ and Leon A. Petrosyan ${ }^{2}$ \\ 1 Institute of Applied Mathematical Research, \\ Karelian Research Center of $R A S$, \\ Pushkinskaya str. 11, Petrozavodsk, 185910, Russia \\ E-mail: annaret@krc.karelia.ru \\ 2 St. Petersburg State University, \\ Faculty of Applied Mathematics and Control Processes, \\ Universitetskii prosp. 35, St. Petersburg, 198504, Russia \\ E-mail: 1.petrosyan@spbu.ru
}

The bibliography is published in connection with the sixty fifth anniversary of professor V.V.Mazalov. Bibliografic data of the scientific papers are presented in the original language.

Professor Mazalov V.V. is widely known specialist in the fields of game theory and operations research. Mazalov V.V. was born in the Urals in the city of Magnitogorsk in 1954. After graduation from school, he entered the faculty of applied mathematics - control processes of the Leningrad State University. He graduated the university in 1976 and continued the post-graduate study with supervision by professor Zubov V.I.

Since 1980, Mazalov V.V. worked at the Chita Institute of Natural Resources of the Siberian Branch of the Russian Academy of Sciences, where he became a director of the Institute (1993-1998). In 1981 Mazalov V.V. defended his candidate thesis on applications of optimal stopping problems and in 1991 - doctoral dissertation "Optimal stopping methods in optimization and minimax problems". Since 1999 he is the organizer and first director of the Institute of Applied Mathematical Research of the Karelian Research Center of the Russian Academy of Sciences.

His first papers were dedicated to the popular Black Jack card game and to the modeling of the best choice problem related to this game.

Mazalov V.V. investigated a wide class of dynamic and evolutionary games that arise in the problems of the ecology of animal behavior and developed methods for finding equilibrium in such games.

Mazalov V.V. proposed new game-theoretic methods in the problems of analyzing the structure of information and communication systems.

Recent studies are related to the generalization of Wardrop equilibrium in transportation problems with rational agents, the construction of stable coalition structures for various payoff distribution procedures, the application of cooperative game theory methods in ranking alternatives in voting procedures and dynamic games with a network structure.

Mazalov prepared $24 \mathrm{Ph} . \mathrm{Dr}$ in mathematics and one doctor of sciences.

The PhD thesis under the supervision of professor Mazalov V.V.:

1. Vinnichenko S.V. "Minimax optimal stopping problems", 1992 (Saint Petersburg)

2. Kovalev S.V. "Risk function in global extremum search problem", 1994 (Saint Petersburg)

3. Kochetov E.A. "Optimal stopping games with incomplete information", 1996 (Saint Petersburg) 
4. Dobrinina N.A. "Zoo-plankton of the Amur basin rivers", 1997 (Irkutsk)

5. Panova S.V. 'Dynamic games with optimal stopping", 1998 (Saint Petersburg)

6. Zabelin A.A. "Bargaining in dynamic games", 2001 (Saint Petersburg)

7. Falko I.A. "House-selling problem", 2001 (Saint Petersburg)

8. Peshkov N.V. "Properties of optimal stopping time in best-choice problem", 2003 (Petrozavodsk)

9. Zhuravlev D.N. "Sequential analysis of the network traffic", 2004 (Petrozavodsk)

10. Rettieva A.N. "Dynamic game methods in the bioresource management problem", 2004 (Petrozavodsk)

11. Chuiko J.V. "Networking games and resource allocation", 2006 (Saint Petersburg)

12. Tokareva J.S. "Equilibrium in arbitration procedures", 2008 (Petrozavodsk)

13. Falko A.A. "N-person best-choice games", 2009 (Saint Petersburg)

14. Kalugina A.M. "Generating functions in voting games", 2009 (Petrozavodsk)

15. Ivashko E.E. "Best choice problems with disorder", 2010 (Saint Petersburg)

16. Truhina L.I. "Cooperative solutions in the networking problems", 2013 (Petrozavodsk)

17. Schiptsova A.V. "Game-theoretic models of resource allocation and applications", 2013 (Petrozavodsk)

18. Plaksina N.V. "Mathematical modelling of passengers flow at the transportation systems", 2014 (Petrozavodsk)

19. Nikitina N.N. "Mathematical models of task allocation and information security in super-computing", 2014 (Petrozavodsk)

20. Noslaskaya T.E. "Stochastic models of bargaining design with many participants", 2014 (Petrozavodsk)

21. Kondratev A.Yu. "Equilibrium in game-theoretic models of bargaining and social choice", 2015 (Saint Petersburg).

22. Tsynguev B.T. 'Mathematical models of ranking of nodes in graphs of communication networks", 2015 (Petrozavodsk)

23. Konovalchikova E.N. "Equilibrium in the best-choice modes with incomplete information", 2016 (Petrozavodsk)

24. Gusev V.V. "Game-theoretic models of search and patrolling in graphs", 2017 (Petrozavodsk)

Also he supervised one Doctor of Science thesis by:

Rettieva A.N. "Cooperation and competition in dynamic bioresource management models", 2016 (Saint Petersburg)

\section{List of publications of V.V.Mazalov}

1. Мазалов В.В. Исследование уравнения для оптимального риска // Вопросы механики и процессов управления, - 1978. - Вып. 2. - с. 129-135.

2. Мазалов В.В., Печников А.А. Об одной задаче оптимизации в информационно-поисковой системе // Автоматизированные системы управления, 1979. - Вып. 2. - с. 28-29. 
3. Мазалов В.В., Соколов А.В. Об оптимальном динамическом распределении нестраничной памяти // Управление в динамических системах, 1979. - c. 206-214.

4. Мазалов В.В. Об играх, определенных на суммах независимых одинаково распределенных случайных величин // Ветвящиеся процессы. Изд-во КФ AH CCCP, - 1981. - c. 30-38.

5. Мазалов В.В. Оптимальный риск и матрица платежей // Вопросы механики и процессов управления, - 1981. - Вып. 4. - с. 160-169.

6. Мазалов В.В. Об оптимальном управлении игровыми случайными последовательностями // Прикладные задачи управления. Изд-во ЛГУ, - 1982. - c. $175-183$.

7. Мазалов В.В. Игровые Марковские моменты // Вероятностные задачи прикладной математики. Изд-во ПГУ, - 1984. - с. 62-68.

8. Мазалов В.В. Игровые моменты остановки. - Новосибирск: Изд-во «Наука», - 1987. 189 с.

9. Мазалов В.В. О некоторых геометрических методах решения задач на правило остановки // Вопросы оптимального управления и исследования операций. Изд-во ИрГУ, - 1988. - с. 120-128.

10. Mazalov V.V., Vinnichenko S.V. Games with a stopping rule for Wiener processes // Theory of Probability and its Applications, - 1989. - Vol. 33 (3). - p. 550-552.

11. Мазалов В.В., Винниченко С.В. Оптимальное управление случайным процессом «Гонка с финишем» // Управление в сложных системах. Изд-во ИвГУ, - 1989. - с. 65-68.

12. Мазалов В.В. Экология и проблемы оптимальной остановки // Математические модели рационального природопользования. Изд-во Наука, 1989. - c. 115-125.

13. Винниченко С.В., Мазалов В.В. Игры оптимальной остановки конечной последовательности наблюдений // Кибернетика, - 1989. - №1. - c. $122-124$.

14. Mazalov V.V., Vinnichenko S.V. Optimal wind choice in races with finish // Approximations, Optimization and Computing (Proceedings of IMACS), Elsevier Sci. Publ, - 1990. - p. 275-279.

15. Mazalov V.V., Vinnichenko S.V. Optimal stopping of observations in control problems of random walks // Theory of Probability and its Applications, - 1991. - Vol. 35 (4). - p. 746-753.

16. Кузин В.Ф., Мазалов В.В., Кердман Ф.С., Фалейчик А.А. Математические методы контроля и управления горным производством. - Иркутск: Изд-во ИрГУ, - 1991. - 216 с.

17. Мазалов В.В., Фалейчик А.А. Математическое моделирование природных структур и процессов // Природные ресурсы Забайкалья. Изд-во ОИГГМ СО РАН СССР, - 1991. - c. 109-127.

18. Mazalov V.V. Choice strategy in optimal control problems for random walks // Probability Theory and Mathematical Statistics. Proceedings of the Sixth USSR-Japan Symposium. World Sci., - 1992. - p. 202-209.

19. Мазалов В.В., Винниченко С.В. Моменты остановки и управляемые случайные блуждания. Новосибирск: Изд-во «Наука», - 1992. - 104 с. 
20. Мазалов В.В., Кондратьев В.А. Управляемый многошаговый процесс с оптимальной остановкой наблюдений // Управление экономическими системами. Изд-во ИвГУ, - 1992. - с. 10-13.

21. Мазалов В.В., Раднаева Д.Б. Оптимизационные задачи на множестве решений уравнений переноса загрязняющих веществ // Моделирование природных систем и задачи оптимального управления. Новосибирск: Изд-во «Наука», - 1993. - с. 18-29.

22. Мазалов В.В., Плюхин Б.В. Численное моделирование проветривания тупиковых выработок // Моделирование природных систем и задачи оптимального управления. Новосибирск: Изд-во «Наука», - 1993. - с. 29-34.

23. Мазалов В.В., Ковалев С.В. Функция риска в задаче планирования эксперимента // Моделирование природных систем и задачи оптимального управления. Новосибирск: Изд-во «Наука», - 1993. - с. 83-92.

24. Мазалов В.В., Домбровский Ю.А., Перрин Н. Теория оптимальной остановки: Приложения к экологии поведения // Обозрение прикладной и промышленной математики, - 1994. - Т. 1, - вып. 6. - с. 893-900.

25. Kovalev S., Mazalov V. A risk function in global optimization problem // Probabilistic methods in discrete mathematics. Proceedings of III International conference in Petrozavodsk, VSP-TVP, Utrecht-Tokyo-Moscow, - 1994. - p. 226-230.

26. Mazalov V.V., Peshkov N.V. Game with selection among two observations of two person // System, Control, Information Methodologies and Applications. Proceedings AMSE SCI"94, Uuhan, China. HUST Press, - 1994. - p. 68-73.

27. Mazalov V.V. A method for Dynamic Allocation of Unpaged Storage // Programming and computer Software, - 1995. - Vol. 21, - no. 4. - p. 183-185.

28. Mazalov V.V. Game-theoretic model of preference // Game Theory and Applications, vol. I. Nova Science Publishers, New York, - 1995. - p. 129-138.

29. Mazalov V.V. The Solution of Russian Black Jack // Game Theory and Applications, vol. I. Nova Science Publishers, New York, - 1995. - p. 41-46.

30. Mazalov V., Kochetov E. Mate choice and optimal stopping problems // Probability Theory and Mathematical Statistics. Proceedings of the Seventh Japan-Russia Symposium, World Sci, - 1995. - p. 317-326.

31. Вотах О.А., Мазалов В.В. (ред.) Окружающая среда и условия устойчивого развития Читинской области. - Новосибирск: Изд-во «Наука», 1995. -248 c.

32. Petrosyan L.A., Mazalov V.V. (eds.). Game Theory and Applications, Vol. I. NY: Nova Science Publishers, - 1995. - 223 p.

33. Mazalov V.V., Perrin N., Dombrovskii Yu.A. Adaptive search and information updating in sequential mate choice // American Naturalist, - 1996. Vol. 148, - no. 1. - p. 123-137.

34. Sakaguchi M., Mazalov V.V. Two-person Hi-Lo Poker-stud and draw // Mathematica Japonica, - 1996. - Vol. 44, - no. 1. - p. 39-53.

35. Mazalov V.V. A game related to optimal stopping of two sequences of independent random variables having different distributions // Mathematica Japonica, - 1996. - Vol. 43, - no. 1. - p. 121-128. 
36. Мазалов В.В., Кочетов Э.А., Перин Н., Панова С.В. Экологические задачи выбора с неполной информацией // Обозрение прикладной и промышленной математики, - 1996. - Т. 3, - вып. 3. - с. 371-383.

37. Mazalov V.V. Dynamic games with optimal stopping // Game theory and Applications, Vol. II. Nova Science Publishers, New York, - 1996. - p. 37-46.

38. Mazalov V.V. Game with Optimal Stopping of Random Walks // Game theory and Applications, Vol. II. Nova Science Publishers, New York, - 1996. p. 161-168.

39. Mazalov V.V., Kochetov E.A. Mate choice and optimal stopping problem // Probability theory and Mathematical Statistics. Proceedings of the VII JapanRussia Simposium. World Scientific, - 1996. - p. 317-326.

40. Petrosyan L.A., Mazalov V.V. (eds.). Game Theory and Applications, Vol. II. NY: Nova Science Publishers, - 1996. -219 p.

41. Mazalov V.V. Method of cumulative sums and problems of optimal control by technological processes // International Journal of Software Engineering and Knowledge Engineering, - 1997. - Vol. 7, - N 2. - p. 219-229.

42. Mazalov V.V., Kochetov E.A. game with optimal stopping of random walks // Theory of Probability and its Applications, - 1998. - Vol. 42 (4). - p. 697701.

43. Mazalov V.V., Sakaguchi M. Two person Hi-Lo poker - stud and draw, II // Game Theory and Applications, Vol. III. Nova Science Publishers, New York, - 1997. - p. 77-90.

44. Mazalov V.V. Stochastic dynamic programming and behavioral ecology problems // Dresdner schriften zur Mathematischen Stochastik, Technische Univ. Dresden, - 1997. - p. 1-18.

45. Petrosyan L.A., Mazalov V.V. (eds.). Game Theory and Applications, Vol. III. NY: Nova Science Publishers, - 1997. - 226 p.

46. Недешев А.А., Мазалов В.В., Винниченко С.В. Факторный анализ географических типов районов Сибири и Дальнего Востока // География и природные ресурсы, - 1998. - № 3. - с. 18-22.

47. Мазалов В.В., Нейман П., Фалько И.А. Игровая задача оптимальной остановки наблюдений с неизвестными значениями // Дальневосточный математический сборник, - 1998. - Вып. 6. - с. 74-86.

48. Mazalov V.V., Kochetov E.A. An optimal stopping of random walks game with reflection // Game Theory and Applications, Vol. IV. Nova Science Publishers, New York, - 1998. - p. 103-109.

49. Petrosyan L.A., Mazalov V.V. (eds.). Game Theory and Applications, Vol. IV. NY: Nova Science Publishers, - 1998. - 194 p.

50. Perrin N., Mazalov V. Dipersal and inbreeding avoidance // American Naturalist, - 1999. - Vol. 154, - no. 3. - p. 282-292.

51. Mazalov V.V., Vdovitsyn V.T., Tarasov V.V. Negotiation on data reallocation in distributed information systems// Proceedings of the 1st International Workshop of Central and Eastern Europe on Multi-Agent Systems CEEMAS‘99. St. Petersburg, - 1999. - p. 47-48

52. Mazalov V.V., Panova S.V., Piskuric M. Two-person bilateral manyrounds poker // Mathematical Methods of Operations Research. - 1999. Vol. $49,-$ iss. 2 . - p. 267-282. 
53. Perrin N., Mazalov V.V. Local competition, inbreeding, and the evolution of sex-biased dispersal // American Naturalist, - 2000. - Vol. 155, - no. 1. p. $116-127$.

54. Kochetov E.A, Mazalov V.V. Geometrical methods for game problems with random walk // Journal of Computer and Systems Sciences International. 2000. - Vol. 39 (4). - p. 545-552.

55. Szajowski K., Kolchin V.F., Kozlov V.Y., Mazalov V.V., Pavlov Y.L., Prokhorov Y.V. On stopping games when more than one stop is possible // Probability methods in discrete mathematics, Proceedings of the Fifth International Petrozavodsk Conference, - 2000. p. 57-72.

56. Petrosyan L.A., Mazalov V.V. (eds.). Game Theory and Applications, Vol. V. NY: Nova Science Publishers, - 2000. - 200 p.

57. Мазалов В.В., Сорокин А.Д., Вдовицын В.Т. Состояние и перспективы формирования электронных информационных ресурсов в КарНЦ РАН для поддержки научных исследований и образования // Материалы конференции "RELARN-2001". Петрозаводск, 2001. - c. 40-42.

58. Mazalov V.V., Makhankov I.S. On a model of two-card poker // Game Theory and Applications, - 2001. - Vol. 7. - p. 115-120.

59. Mazalov V.V., Zabelin A.A. Non-symmetric solution of an arbitration game // Game Theory and Applications, - 2001. - Vol. 7. - p. 115-120.

60. Леймар О., Мазалов В.В. Бета-распределение в одной эволюционной игре // Обозрение прикладной и промышленной математики, -2001 . - T. 8, - вып. 1. - с. 251-252.

61. Перрин Н., Мазалов В.В. Методы теории восстановления в задачах оптимального добывания пищи // Журнал общей биологии, - 2001. - Т. 62, 一 №2. - c. 171-179.

62. Petrosyan L.A., Mazalov V.V. (eds.). Game Theory and Applications, Vol. VI. NY: Nova Science Publishers, - 2001. - 206 p.

63. Petrosyan L.A., Mazalov V.V. (eds.). Game Theory and Applications, Vol. VII. NY: Nova Science Publishers, - 2001. - 202 p.

64. Mazalov V.V., Rettieva A.N. Reserved territory approach for a management problem with distributed resource // Proc. of the International Congress of Mathematicians 2002. Satellite Conference on GTA, - 2002. - p. 493-499.

65. Mazalov V.V., Leimar O. Coin tossing and game of attrition // Proc. of the International Congress of Mathematicians 2002. Satellite Conference on GTA, - 2002. - p. 487-492.

66. Mazalov V.V., Zabelin A.A. Discrete final-offer arbitration model // Development of the Optimization Theory for the Dynamic Systems and their Applications, RIMS seria. Kyoto University, - 2002. - Vol. 1263. - p. 117-130.

67. Mazalov V.V., Zabelin A.A., Karpin A.A. Equilibrium in an arbitration game / Probabilistic Methods in Discrete Mathematics. Proc. of V Int. Petrozavodsk Conf., VSP, -2002 . - p. 41-46.

68. Mazalov V.V., Zabelin A.A. Multistage arbitration game with random offers // Proc. of X Int. Symposium on Dynamic Games and Applications, StPetersburg University Press, - 2002. - Vol. 2. - p. 578-581. 
69. Mazalov V.V., Sakaguchi M., Zabelin A.A. Multistage arbitration game with random offers // Game Theory and Applications, - 2002. - Vol. 8. - p. 95-106.

70. Mazalov V.V., Rettieva A.N. On a reserved territory approach for a resource management problem // Proc. of X Int. Symposium on Dynamic Games and Applications, St-Petersburg University Press, - 2002. - Vol. 2. - p. 575-577.

71. Mazalov V.V., Saario V. The house-selling problem with reward rate criterion // Journal of Applied Probability, - 2002. - Vol. 39, - no. 3. - p. 644-649.

72. Mazalov V.V, Zhuravlev D.N. A method of cumulative sums in the problem of detection of traffic changes in computer networks // Programming and Computer Software, - 2002. - Vol. 28 (6). - p. 342-348.

73. Мазалов В.В., Реттиева А.Н. Об одной задаче управления биоресурсами // Обозрение прикладной и промышленной математики, - 2002. - Т. 9, - вып. 2. - с. 293-306.

74. Petrosyan L.A., Mazalov V.V. (eds.). Game Theory and Applications, Vol. 8. NY: Nova Science Publishers, - 2002. - 295 p.

75. Mazalov V., Sakaguchi M. Location game on the plane // International Game Theory Review, - 2003. - Vol. 5, - N 1. - p. 13-25.

76. Mazalov V.V., Banin M.V. N-person best-choice game with voting // Game Theory and Applications, - 2003. - Vol. 9. - p. 45-54.

77. Mazalov V.V., Rettieva A.N. A fishery game model with age-distributed population: Reserved territory approach // Game Theory and Applications, 2003. - Vol. 9. - p. 55-70.

78. Mazalov V., Tamaki M. Explicit solutions to the duration problem // Aichi Keiei Ronsyu, - 2003. - Vol. 147. - p. 69-92.

79. Petrosyan L.A., Mazalov V.V. (eds.). Game Theory and Applications, Vol. 9. NY: Nova Science Publishers, - 2003. - 263 p.

80. Mazalov V.V., Banin M.V., Sakaguchi M. Sequential arbitration games // Proceedings of 11-th Int. Symposium on Dynamic Games and Applications, Univ. of Arizona, - 2004. - Vol. 2. - p. 615-624.

81. Sakaguchi M., Mazalov V.V. A non-zero-sum no-information best-choice game // Math. methods of operations research, -2004 . - V. 60, - no. 3. - p. 437-451.

82. Mazalov V.V., Rettieva A.N. Reserved territory approach in a fishery game model // Proceedings of 11-th Int. Symposium on Dynamic Games and Applications, Univ. of Arizona, - 2004. - Vol. 1. - p. 603-614.

83. Mazalov V.V., Rettieva A.N. A fishery game model with migration: Reserved territory approach // Game Theory and Applications, - 2004. - Vol. 10. - p. 97-108.

84. Mazalov V.V., Rettieva A.N. A Fishery Game Model with Migration: Reserved Territory Approach // Труды 4-й Московской международной конференции по исследованию операций (ORM2004). М.: MAKC Пресс, - 2004. - c. $151-154$.

85. Банин М.В., Мазалов В.В. Асимптотические свойства оптимального выигрыша в задаче переговоров с голосованием // Методы математическо- 
го моделирования и информационные технологии. Труды ИПМИ. Петрозаводск: КарНЦ РАН, - 2004. - Вып. 5. - с. 13-18.

86. Mazalov V.V., Peshkov N.V. On asymptotic properties of optimal stopping time // Theory of Probability and its Applications, - 2004. - Vol. 48 (3). p. 549-555.

87. Mazalov V.V., Zabelin A.A. Equilibrium in an arbitration procedure // Annals of Dynamic Games, - 2004. - Vol. 7. - p. 295-305.

88. Мазалов В.В., Пешков Н.В. Асимптотические свойства оптимального момента остановки в задаче с ненулевой платой за наблюдения // Вестник СПбГУ, сер. 10, - 2005. - Вып. 2. с. 146-150.

89. Мазалов В.В., Реттиева А.Н. Методы динамических игр в задаче определения оптимальной заповедной зоны // Обозрение прикладной и промышленной математики, - 2005. - Т. 12, - вып. 3. - с. 610-625.

90. Mazalov V.V., Zabelin A.A. Equilibrium in an arbitration procedure // Advances in dynamic Games, - 2005. - Vol. 7. - p. 295-305.

91. Petrosyan L.A., Mazalov V.V. (eds.). Game Theory and Applications, Vol. 10. NY: Nova Science Publishers, -2005 . -235 p.

92. Mazalov V., Tamaki M. An explicit formula for the optimal gain in the fullinformation problem of owning a relatively best object // Journal of Applied Probability, - 2006. - Vol. 43, - no. 1. - p. 87-101.

93. Mazalov V.V., Mentcher A.E., Tokareva J.S. On a discrete arbitration procedure in three points // Game Theory and Applications, - 2006. - Vol. 11. - p. 87-91.

94. Mazalov V.V., Rettieva A.N. Nash equilibrium in bioresource management problem with changing area for fishery // Natural Resources and the Environment. Full text of the presentations of 6th Meeting on Game Theory and Practice Dedicated to Development. IAMZ, Zaragoza, Spain, - 2006.

95. Mazalov V., Mentcher A., Tokareva J. On a discrete arbitration procedure // Scientiae Mathematicae Japonicae, - 2006. - Vol. 63, - no.3. - p. 325-330.

96. Mazalov V., Monien B., Schoppmann F., Tiemann K. Wardrop Equilibria and Price of Stability for Bottleneck Games with Splittable Traffic // 2nd international Workshop on Internet \& Network Economics (WINE 2006, Patras / Greece). Lecture Notes in Computer Science, - 2006. - Vol. 4286. p. 331-342.

97. Мазалов В.В., Фалько А.А. Голосование в задаче наилучшего выбора с ранговым критерием // Обозрение прикладной и промышленной математики, - 2006. - Т. 13, - № 4. - с. 577-588.

98. Мазалов В.В., Реттиева А.А., Родионов А.В., Цыпук А.М., Шишкин А.И. Моделирование экономических отношений в лесном комплексе Республики Карелия // Труды КарНЦ РАН. Петрозаводск: КарНЦ РАН, - 2006. - Вып. 9. - с. 144-154.

99. Вдовицын В.Т., Луговая Н.Б., Мазалов В.В., Чуйко Ю.В. Применение теоретико-игрового подхода для оптимального использования ресурсов сети передачи данных // Научный сервис в сети Интернет: технологии параллельного программирования. Труды Всерос. научной конф. М.: Изд-во МГУ, - 2006. - с. 207-208. 
100. Мазалов В.В., Реттиева А.Н. Равновесие по Нэшу в задачах охраны окружающей среды // Математическое моделирование, - 2006. - Т. 18, № 5. - c. $73-90$.

101. Sakaguchi M., Mazalov V. Equilibrium in N-player competitive silent games of timing // Game Theory and Applications, - 2006. - Vol. 12. - p. 143-156.

102. Мазалов В.В., Чуйко Ю.В. Некооперативное равновесие по Нэшу в задаче выбора оптимального момента обращения к системе обслуживания // Вычислительные технологии, - 2006. - Т. 11, - №6. - c. 60-71.

103. Мазалов В.В., Сорокин А.Д. Институт прикладных математических исследований // Академическая наука в Карелии: 1946-2006. М: Наука, 2006. - T. 2. - c. 300-326.

104. Mazalov V.V., Rettieva A.N. Cooperative incentive equilibrium for a bioresource management problem // Contributions to Game Theory and Management. Collected papers of the Int. Conference "Game Theory and Management". St.Petersburg University, - 2007. p. 316-325.

105. Mazalov V.V., Tamaki M. Duration problem on trajectories // Stochastics: An International Journal of Probability and Stochastic Processes, - 2007. Vol. 79, - no. 3-4. - p. 211-218.

106. Mazalov V., Tokareva J. Equilibrium in combined arbitration procedure // Proc. II International Conf. in Game Theory and Applications, Qingdao, China, - 2007. - p. 186-188.

107. Sakaguchi M., Mazalov V.V. Equilibrium in n-player competitive silent games of timing // Game Theory and Applications, - 2007. - Vol. 12. - p. 153-166.

108. Мазалов В.В., Фалько А.А. Задача наилучшего выбора и ее применение в рекламных кампаниях поисковой системы Яндекс // ИнтернетМатематика 2007. Яндекс, - 2007. - с. 126-134.

109. Мазалов В.В., Ивашко Е.Е. Задача наилучшего выбора с разладкой // Обозрение прикладной и промышленной математики, - 2007. - T. 14, вып. 2. - с. 215-224.

110. Мазалов В.В., Чуйко Ю.В. Справедливое разделение пропускной способности каналов сети // Вестник СПбГУ. Серия 10, - 2007. - Вып. 2. c. $42-54$.

111. Ширяев А.Н., Мазалов В.В. Российско-скандинавский симпозиум «Терия вероятностей и прикладная вероятность» // Теория вероятностей и ее применения, - 2007. - Вып. 52 (1). - с. 204-205.

112. Petrosyan L.A., Mazalov V.V. (eds.). Game Theory and Applications, Vol. 11. NY: Nova Science Publishers, - 2007. - 227 p.

113. Petrosyan L.A., Mazalov V.V. (eds.). Game Theory and Applications, Vol. 12. NY: Nova Science Publishers, - 2007. - 248 p.

114. Mazalov V.V., Tokareva J.S. Bargaining model on the plane// Algorithmic and Computational Theory in Algebra and Languages. RIMS Kokyuroky 1604. Kyoto University, - 2008. - p. 42-49.

115. Mazalov V.V., Rettieva A.N. Bioresource management problem with changing area for fishery // Game Theory and Applications, - 2008. - Vol. 13. - p. $101-110$. 
116. Mazalov V.V., Falko I.A., Gurtov A.V., Pechnikov A.A. Equilibrium in P2P-system // Proceedings of AMICT'2007. Annual International Workshop, Petrozavodsk State University, - 2008. - Vol. 9. - p. 129-139.

117. Mazalov V.V., Rettieva A.N. Incentive equilibrium in bioresource management problem // Evolutionary methods for design, optimization and control, CIMNE, Barcelona, Spain, - 2008. p. 301-312.

118. Chuiko J.V., Mazalov V.V. Nash Equilibrium in Splittable Traffic Routing Problem // Proceedings of Kosen Workshop MTE2008 - Mathematics, Technology and Education - Ibaraki National College of Thechnology, Hitachinaka, Ibaraki, Japan, - 2008. - p. 13-18.

119. Mazalov V.V., Falko A.A. Nash equilibrium in two-sided mate choice problem // International Game Theory Review. - 2008. - Vol. 10, - no. 4. - p. 421-435.

120. Мазалов В.В., Фалько А.А. Арбитражная процедура в задаче совместного наилучшего выбора для т лиц // Вестник СПбГУ. Серия 10, 2008. - Вып. 4. - c. 52-59.

121. Mazalov V.V., Falko A.A. House-selling problem with reward rate criteria and changing costs // Journal of Computer and Systems Sciences International, - 2008. - Vol. 47. - N 2. - p. 235-244.

122. Мазалов В.В., Тамаки М., Винниченко С.В. Оптимальное распределение памяти компьютера для пуассоновских потоков // Автоматика и телемеханика, - 2008. - N 9. - c. 69-75.

123. Mazalov V.V., Rettieva A.N. Incentive equilibrium in discrete-time bioresource sharing model // Doklady Mathematics, - 2008. - Vol. 78 (3). - p. 953-955.

124. Mazalov V.V., Rettieva A.N. A Fishery Game Model with Migration: Reserved Territory Approach // Mathematics, Game Theory and Algebra Compendium, - 2009. - Vol. 1. - p. 283-299.

125. Petrosyan L.A., Mazalov V.V. (eds.). Game Theory and Applications, Vol. 13. NY: Nova Science Publishers, - 2008. - $231 \mathrm{p}$.

126. Sandkuhl K., Smirnov A., Mazalov V., Vdovitsyn V., Tarasov V., Krizhanovsky A., Lin F., Ivashko E. Context-Based Retrieval in Digital Libraries: Approach and Technological Framework // Электронные библиотеки: перспективные методы и технологии, электронные коллекции: Труды XI Всероссийской научной конференции RCDL'2009. Петрозаводск: КарНЦ PAH, - 2009. - c. 151-157.

127. Lundqvist M., Mazalov V., Sandkuhl K., Vdovitsyn V., Ivashko E. Do Digital Libraries satisfy Users' Information Demand? Findings from an Empirical Study // Электронные библиотеки: перспективные методы и технологии, электронные коллекции: Труды ХI Всероссийской научной конференции RCDL'2009. Петрозаводск: КарНЦ РАН, - 2009. - c. 167-174.

128. Mazalov V.V., Falko A.A. Mutual mate choice problem with Arrivals // Contributions to Game theory and management. Collected papers presented on the Second International Conference Game Theory and Management. SPb.: Graduate Scholl of Management, - 2009. — p. 271-280. 
129. Mazalov V.V., Rettieva A.N. The compleat fish wars with changing area for fishery // IFAC Proceedings Volumes (IFAC-PapersOnLine) , - 2009. Vol. 7 (1). - p. 168-172.

130. Мазалов В.В., Ивашко Е.Е. Байесовская модель в задаче наилучшего выбора с «разладкой» // Вестник СПбГУ, Сер. 10, - 2009. - Вып. 4. - с. $142-151$.

131. Мазалов В.В., Ивашко Е.Е. Задача выбора оптимального объема запрашиваемых ресурсов в условиях ожидаемой «разладки» сервера / Системы управления и информационные технологии, -2009 . - №1.2(35). - c. 249252.

132. Mazalov V.V., Mencher A.E., Tokareva Yu.S. On the equilibrium in bargaining model with arbitrator // Journal of Computer and Systems Sciences International, - 2009. - Vol. 48, - no. 5. - p. 739-745.

133. Мазалов В.В., Печников А.А. О рейтинге официальных сайтов научных учреждений северо-запада России // Управление большими системами. М: ИПУ РАН, - 2009. - Вып. 24. - с. 130-146.

134. Мазалов В.В., Печников А.А., Фалько И.А. О построении рейтинга научных журналов // Управление большими системами, - 2009. - Вып. 27. - c. $47-52$.

135. Мазалов В.В., Сакагучи М. Равновесие в бескоалиционной игре n лиц с выбором момента времени // Управление большими системами, - 2009. - Вып. 26.1. - с. 55-78.

136. Sandkuhl K., Tarasov V., Mazalov V., Smirnov A. Context based Retrieval in Digital Libraries (CoReLib): Final project report // School of Engineering at Jöonköping University, - 2009.

137. Petrosyan L.A., Mazalov V.V. (eds.). Game Theory and Applications, Vol. 14. NY: Nova Science Publishers, - 2009. - 191 p.

138. Мазалов В.В. Математическая теория игр и приложения. Санкт-ПетербургМосква-Краснодар, Лань, - 2010. - 446 с.

139. Mazalov V.V., Falko A.A. Equilibrium in n-person game of Showcase-Showdown // Probability in the Engineering and Informational Sciences, Cambridge Univ. Press, - 2010. - Vol. 24. - p. 397-403.

140. Mazalov V.V., Rettieva A.N. Fish wars and cooperation maintenance // Ecological Modelling, - 2010. - Vol. 221. - p. 1545-1553.

141. Mazalov V.V., Rettieva A.N. Fish wars with many players // International Game Theory Review, - 2010. - Vol. 12, - issue 4. - p. 385-405.

142. Мазалов В.В., Щипцова А.В., Токарева Ю.С. Дуополия Хотеллинга и задача о размещении на плоскости // Экономика и математические методы, - 2010. - Т. 46, - вып. 4. - с. 91-100.

143. Мазалов В.В., Печников А.А., Чирков А.В., Чуйко Ю.В. Задача дележа затрат на создание веб-коммуникатора как кооперативная игра // Управление большими системами, - 2010. - Вып. 30.1. - с. 187-196.

144. Mazalov V.V., Rettieva A.N. Incentive equilibrium in bioresource sharing problem // Journal of Computer and Systems Sciences International, - 2010.

- Vol. 49 (4). - p. 598-606. 
145. Мазалов В.В., Токарева Ю.С. Теоретико-игровые модели проведения конкурсов // Математическая Теория Игр и ее Приложения, -2010 . - Т. 2, - вып. 2. - с. 66-78.

146. Mazalov V.V., Rettieva A.N. Incentive conditions for rational behavior in discrete-time bioresource management problem // Doklady Mathematics, 2010. - Vol. 81 (3). - p. 399-402.

147. Lukyanenko A.S., Gurtov A.V., Mazalov V.V. Applying a reputation metric in a two-player resource sharing game // Contributions to Game theory and management. Collected papers presented on the Third Int. Conference Game Theory and Management. SPb.: Graduate Scholl of Management, - 2010. - p. 182-191.

148. Мазалов В.В., Токарева Ю.С. Теоретико-игровые модели проведения конкурсов // Управление большими системами, - 2010. - Вып. 31. - 273286.

149. Ширяев А.Н., Мазалов В.В. Международная конференция «Стохастическая теория оптимальной остановки» // Теория вероятностей и ее применения, - 2010. - Вып. 55 (4). - с. 823-824.

150. Токарева Ю.С., Мазалов В.В. Эффект корреляции в модели переговоров с арбитражным комитетом // Математический анализ и его приложения, $-2010 .-$ c.49-54.

151. Lukyanenko A., Mazalov V., Gurtov A., Falko I. Playing Defense by Offense: Equilibrium in the DoS-attack problem // The IEEE symposium on Computers and Communications, - 2010. - p. 433-436.

152. Mazalov V., Ivashko E. Bayes' Model of the Best-Choice Problem with Disorder // International Journal of Stochastic Analysis, - 2011. - Vol. 2012.

153. Mazalov V.V., Rettieva A.N. Cooperation Maintenance in Fishery Problems // Fishery Management. Nova Science Publishers, - 2011. - p. 151-198.

154. Мазалов В.В., Трухина Л.И. $n$-ядро и распределение памяти компьютера // Вычислительные технологии, - 2011. - Т. 16, - №6. - c. 48-54.

155. Mazalov V.V., Rettieva A.N. The discrete-time bioresource sharing model // Journal of Applied Mathematics and Mechanics, - 2011. - Vol. 75(2). - p. 180-188.

156. Мазалов В.В., Ивашко А.А. Международная конференция « Стохастическая теория оптимальной остановки» (Петрозаводск, 12-16 сентября 2010 г.) // Труды КарНЦ РАН. Сер. Математическое моделирование и информационные технологии, - 2011. - Вып. 2. - с. 130-131.

157. Мазалов В.В., Токарева Ю.С. Равновесие в задаче о сделках с неравномерным распределением резервных цен // Математическая Теория Игр и ее Приложения, - 2011. - Т. 3, - вып. 2. - с. 37-49.

158. Мазалов В.В., Токарева Ю.С. Равновесие в сделках с фиксированными ценами // Ученые записки Забайкальского государственного гуманитарнопедагогического университета, - 2011. - Вып.3. - с. 103-107.

159. Petrosyan L.A., Mazalov V.V. (eds.). Game Theory and Applications, Vol. 15. NY: Nova Science Publishers, $-2011 .-225$ p. 
160. Mititelu G., Mazalov V.V., Novikov A. On CUSUM procedure for hyperexponential distribution (working paper).

161. Мазалов В.В., Менчер А.Э., Токарева Ю.С. Переговоры. Математическая теория. Санкт-Петербург-Москва-Краснодар, Лань, - 2012. - 304 c.

162. Chuiko J.V., Mazalov V.V., Polishchuk T.O., Gurtov A.V. Wardrop equilibria and price of anarchy in multipath routing games with tcp traffic // International Journal of Mathematics, Game Theory, and Algebra, - 2012. Vol. 20 (4). - p. 263.

163. Mazalov V., Tokareva Yu. Arbitration procedures with multiple arbitrators // European Journal of Operational Research, - 2012. - Vol. 217, - issue 1. - p. 198-203.

164. Mazalov V., Gurtov A. Queueing System with On-Demand Number of Servers // Mathematica Applicanda, - 2012. - Vol. 40 (2). - p. 1-12.

165. Chuyko J., Polishchuk T., Mazalov V., Gurtov A. Wardrop Equilibria and Price of Anarchy in Multipath Routing Games with Elastic Traffic // Game Theory and Applications. - 2012. - Vol. 13. - p. 9-19.

166. Мазалов В.В., Кондратьев А.Ю. Задача о сделках с неполной информацией // Вестник Санкт-Петербургского университета. Серия 10: Прикладная математика. Информатика. Процессы управления, - 2012. - №1. - c. $33-40$.

167. Мазалов В.В., Никитина Н.Н. Оценка характеристик алгоритма Backfill при управлении потоком задач на вычислительном кластере // Вычислительные технологии, - 2012. - Т. 17, - № 5. - с. 71-79.

168. Мазалов В.В., Токарева Ю.С. Репутации арбитров в моделях проведения переговоров // Труды КарНЦ РАН. Сер. Математическое моделирование и информационные технологии, -2012 . - Вып. 3. - с. 61-67.

169. Мазалов В.В., Носальская Т.Э. Стохастический дизайн в задаче о дележе пирога // Математическая Теория Игр и ее Приложения, - 2012. T. 4, - вып. 3. - с. 33-50.

170. Mazalov V., Ivashko E. Bayes' Model of the Best-Choice Problem with Disorder // International Journal of Stochastic Analysis. - 2012.

171. Sandkuhl K., Tarasov V., Mazalov V., Smirnov A. Context based Retrieval in Digital Libraries: Continuing PhD Education and Experimentation (CoReLib2): Final project report J?nk?ping University, School of Engineering, $-2012$.

172. Ou Z., Zhuang H., Lukyanenko A., Nurminen J., Hui P., Mazalov V., Yla-Jaaski A. Is the Same Instance Type Created Equal? Exploiting Heterogeneity of Public Clouds // IEEE Transactions on Cloud Computing, 2013. - Vol. PP, - issue 99. - p. 14.

173. Токарева Ю.С., Мазалов В.В. Об одной задаче наилучшего выбора с правилом консенсуса // Ученые Записки ЗабГУ, - 2013. - Вып. 3(50). c. $105-109$.

174. Мазалов В.В., Кондратьев А.Ю. Равновесие в сделках с пороговыми стратегиями // Математическая Теория Игр и ее Приложения, - 2013. Т. 5, - вып. 2. - с. 46-63. 
175. Petrosyan L.A., Mazalov V.V. (eds.). Game Theory and Applications, Vol. 16. NY: Nova Science Publishers, - 2013. - 222 p.

176. Mazalov V.V. (ed.). NGM-2013. Networking Games and Management: International workshop. Petrozavodsk: KRC RAS, $-2013 .-106 \mathrm{p}$.

177. Mazalov V. Mathematical Game Theory and Applications. John Wiley \& Sons, -2014 . $-414 \mathrm{p}$.

178. Мазалов В.В., Реттиева А.Н. Теоретико-игровые модели кооперации в задачах управления биоресурсами // Модели и методы в проблеме взаимодействия атмосферы и гидросферы: учебное пособие. Томск: изд-во ТГУ, - 2014. - c. 449-489.

179. Mazalov V.V., Nikitina N.N. A CUSUM Method to Detect and Counteract Intrusions // Programming and Computer Software, - 2014. - Vol. 40, - issue 6. - p. 337-345.

180. Mazalov V.V., Tokareva J.S. Game-theoretic models of tender design // Automation and Remote Control, - 2014. - Vol. 75, - issue 10. - p. 18481860.

181. Mazalov V.V., Nikitina N.N., Ivashko E.E. Hierarchical Two-Level Game Model for Tasks Scheduling in a Desktop Grid // Applied Problems in Theory of Probabilities and Mathematical Statistics Related to Modeling of Information Systems, - 2014. - p. 641-645.

182. Gao H., Hu J., Mazalov V., Shchiptsova A., Song L., Tokareva J. Location-Price Competition in Airline Networks // Journal of Applied Mathematics, - 2014. - Vol. 2014.

183. Gao H., Hu J., Mazalov V., Shchiptsova A., Song L., Tokareva J. Location-price Game-theoretic Model and Applications in Transportation Networks // Procedia Computer Science, — 2014. - Vol. 31. - p. 754-757.

184. Mazalov V., Nosalskaya T., Tokareva J. Stochastic Cake Division Protocol // International Game Theory Review, - 2014. - Vol. 16, - issue 2.

185. Буре В.М., Мазалов В.В., Плаксина Н.В. Вычисление характеристик пассажиропотоков в транспортных системах // Управление большими системами, - 2014. - Вып. 47. - с. 77-91.

186. Мазалов В.В., Чиркова Ю.В., Ивашко А.А. Международное рабочее совещание «Сетевые игры и менеджмент» (Петрозаводск, 23-25 июня 2013 г.) // Труды КарНЦ РАН. Сер. Математическое моделирование и информационные технологии, - 2014. - №4. - c. 168-169.

187. Mazalov V.V., Trukhina L.I. Generating functions and the Myerson vector in communication networks // Discrete Mathematics and Applications, - 2014. - Vol. 24 (5). - p. 295-303.

188. Гао Х., Мазалов В.В., Ху Ц., Щипцова А.В. Равновесие в игре размещения на рынке авиаперевозок // Труды КарНЦ РАН. Сер. Математическое моделирование и информационные технологии, -2014 . - № 4. - c. $41-47$.

189. Ивашко А.А., Коновальчикова Е.Н., Мазалов В.В. Теоретико-игровые иерархические модели выбора // Труды XII Всероссийского совещания по проблемам управления ВСПУ-2014. Москва: ИПУ РАН, - 2014. - c. 83088313. 
190. Mazalov V.V., Rettieva A.N. Chapter 8. Assymetry in a cooperative bioresource management problem // Game-Theoretic Models in Mathematical Ecology. NY: Nova Science Publishers, - 2015. - p. 113-152.

191. Avrachenkov K.E., Mazalov V.V., Tsynguev B.T. Beta Current Flow Centrality for Weighted Networks // Computational Social Networks. Lecture Notes in Computer Science, - 2015. - Vol. 9197. - p. 216-227.

192. Mazalov V, Lukyanenko A., Luukkainen S. Equilibrium in cloud computing market // Performance Evaluation, - 2015. - Vol. 92. - p. 40-50.

193. Bure V.M., Mazalov V.V., Plaksina N.V. Estimating passenger traffic characteristics in transport systems // Automation and Remote Control, 2015. - Vol. 76, - issue 9. - p. 1673-1680.

194. Bure V.M., Mazalov V.V., Melnik A.V., Plaksina N.V. Passenger Traffic Evaluation and Price Formation on the Transportation Services Market // Advances in Operations Research, - 2015. - Vol. 2015.

195. Mazalov V.V., Nikitina N.N., Ivashko E.E. Task Scheduling in a Desktop Grid to Minimize the Server Load // Lecture Notes in Computer Science. 13th International Conference on Parallel Computing Technologies, PaCT 2015, 2015. - Vol. 9251. p. 273-278.

196. Mazalov V.V., Kondratev A.Y. The bargaining solution among threshold strategies // Automation and Remote Control, - 2015. - Vol. 76, - issue 3. p. $507-520$.

197. Mazalov V., Konovalchikova E. The job-search problem with incomplete information // Procedia Computer Science, - 2015. - Vol. 55. - p. 159-164.

198. Petrosyan L.A., Mazalov V.V. (eds.). Game Theory and Applications, Vol. 17. NY: Nova Science Publishers, - 2015. - 234 p.

199. Мазалов В.В., Реттиева А.Н. Асимметрия в кооперативной задаче управления биоресурсами // Управление большими системами, - 2015. - Вып. 55. - c. $280-325$.

200. Гусев В.В., Мазалов В.В. Оптимальные стратегии в игре патрулирования на графе // Вестник С.-Петербург. ун-та. Сер. 10. Прикл. матем. информ. проц. упр., - 2015. - №2. - с. 61-76.

201. Жижченко А.Б., Мазалов В.В., Цынгуев Б.Т. Ранжирование вершин графа публикаций математического портала Math-Net.ru // Труды КарНЦ РАН. Сер. Математическое моделирование и информационные технологии, - 2015. 一 №10. - c. 34-41.

202. Коновальчикова Е.Н., Мазалов В.В. Теоретико-игровая модель телевизионного конкурса «Голос» // Математическая Теория Игр и ее Приложения, - 2015. - Т. 7, - вып. 2. - с. 14-32.

203. Мазалов В.В., Ивашко А.А. Третий Российско-Финский симпозиум по дискретной математике (Петрозаводск, 15 - 18 сентября 2014 г.) // Труды КарНЦ РАН. Сер. Математическое моделирование и информационные технологии, - 2015. - №10. - с. 145-146.

204. Мазалов В.В. Математическая теория игр и приложения (второе издание). Санкт-Петербург-Москва-Краснодар: Лань, - 2016. - 446 с.

205. Konovalchikova E.N., Mazalov V.V. A Game-Theoretic Model of TV Show "The Voice" // Automation and Remote Control, - 2016. - Vol. 77, - issue 8. - p. $1468-1479$. 
206. Mazalov V.V., Melnik A.V. Equilibrium Prices and Flows in the Passenger Traffic Problem // International Game Theory Review, - 2016. - Vol. 18, no. 1.

207. Mazalov V.V., Avrachenkov K.E., Trukhina L.I., Tsynguev B.T. GameTheoretic Centrality Measures for Weighted Graphs // Fundamenta Informaticae, - 2016. - Vol. 145, - issue 3. - p. 341-358.

208. Mazalov V.V. , Tsynguev B.T. Kirchhoff Centrality Measure for Collaboration Network // Computational Social Networks. Lecture Notes in Computer Science, - 2016. - Vol. 9791. - p. 147-157.

209. Mazalov V., Shchiptsova A., Tokareva Yu. Location Game and Applications in Transportation Networks // Lecture Notes in Economics and Mathematical Systems. Computational Management Science, - 2016. - Vol. 682. p. 55-61.

210. Zhang G., Hu J., Wang L., Sun H., Mazalov V. Multi-period closedloop supply chain network equilibrium with the consumer's preference for trade channels // Systems Engineering - Theory \& Practice, - 2016. - Vol. 36, no. 2. - p. 347-362.

211. Mazalov V.V., Ivashko A.A., Konovalchikova E.N. Optimal Strategies in Best-Choice Game with Incomplete Information - The Voice Show // International Game Theory Review, - 2016. - Vol. 18, - no. 2.

212. Lien J.W., Mazalov V.V., Melnik A.V., Zheng J. Wardrop Equilibrium for Networks with the BPR Latency Function // Discrete Optimization and Operations Research. Lecture Notes in Computer Science, - 2016. - Vol. 9869. - p. 37-49.

213. Мазалов В.В., Печников А.А., Трухина Л.И., Цынгуев Б.Т. Ранжирование академического веб-пространства // Управление большими системами, - 2016. - Вып. 61. - с. 118-135.

214. Karhumaki J., Mazalov V., Matiyasevich Y. Discrete Mathematics (RuFiDiM 14) Preface // Fundamenta Informaticae, - 2016. - Vol. 145 (3). p. I-II.

215. Petrosyan L.A., Mazalov V.V. (eds.). Recent Advances in Game Theory and Applications. European Meeting on Game Theory, Saint Petersburg, Russia, 2015, and Networking Games and Management, Petrozavodsk, Russia, 2015. Birkhauser, - 2016. - 284 p.

216. Мазалов В.В. Математическая теория игр и приложения (третье издание). Санкт-Петербург-Москва-Краснодар: Лань, - 2017. - 446 с.

217. Petrosyan L.A., Mazalov V.V., Zenkevich, N.A. (eds.). Frontiers of Dynamic Games: Game Theory and Management, St. Petersburg, 2017. Springer, - 2018. - $282 \mathrm{p}$.

218. Kondratev A., Mazalov V. A Ranking Procedure with the Shapley Value // Intelligent Information and Database Systems. ACIIDS 2017. Lecture Notes in Computer Science, - 2017. - Vol. 10192. - p. 691-700.

219. Avrachenkov K.E., Kondratev A.Yu., Mazalov V.V. Cooperative Game Theory Approaches for Network Partitioning // COCOON 2017. Lecture Notes in Computer Science, - 2017. - Vol. 10392. - p. 591-603. 
220. Mazalov V.V., Rettieva A.N., Avrachenkov K.E. Linear-quadratic discretetime dynamic potential games // Automation and Remote Control, - 2017. Vol. 78, - issue 8. - p. 1537-1544.

221. Kondratev, A.Y., Mazalov, V.V. Nucleolus as Tournament Solution // Proceedings of the Fourth Russian Finnish Symposium on Discrete Mathematics, - 2017. - p. 93-95.

222. Мазалов В.В., Реттиева А.Н., Авраченков К.Е. Линейно-квадратичные динамические потенциальные игры в дискретном времени // Математическая Теория Игр и ее Приложения, - 2017. - Т. 9, - вып. 1. - с. $95-107$.

223. Мазалов В.В., Чиркова Ю.В., Зенг Д., Лиен Д. Теоретико-игровая модель поведения конкурирующих виртуальных операторов на двухстороннем рынке телекоммуникаций // Математическая Теория Игр и ее Приложения, - 2017. - Т. 9, - вып. 3. - с. 36-63.

224. Ермолин Н.А., Мазалов В.В., Печников А.А. Теоретико-игровые методы нахождения сообществ в академическом Вебе // Труды СПИИРАН, - 2017. - Вып. 55. - с. 237-254.

225. Мазалов В.В., Чиркова Ю.В. Сетевые игры. Изд-во Лань, - 2018. $320 \mathrm{c}$.

226. Mazalov V.V., Chirkova Yu.V., Zheng J., Lien J.W. A Game-Theoretic Model of Virtual Operators Competition in a Two-Sided Telecommunication Market // Automation and Remote Control, - 2018. - Vol. 79, - issue 4. p. $737-756$.

227. Mazalov V.V. Comparing Game-Theoretic and Maximum Likelihood Approaches for Network Partitioning // Transactions on Computational Collective Intelligence, XXXI, LNCS, - 2018. - Vol. 11290. - p. 37-46.

228. Avrachenkov K.E., Kondratev A.E., Mazalov V.V., Rubanov D.G. Network partitioning algorithms as cooperative games // Computational Social Networks, - 2018. - Vol. 5:11. - p. 1-28.

229. Мазалов В.В., Никитина Н.Н. Метод максимального правдоподобия для выделения сообществ в коммуникационных сетях // Вестник СанктПетербургского университета. Прикладная математика. Информатика. Процессы управления, -2018 . - Т. 14, - вып. 3. - с. 200-214.

230. Гусев В.В., Мазалов В.В. Устойчивые по Оуэну коалиционные разбиения в играх с векторными платежами // Математическая Теория Игр и ее Приложения, - 2018. - Т. 10, - вып. 3. - с. 3-23.

231. Фенг Д., Лиу Т., Мазалов В.В., Зенг Д. Ценообразование на сетевых платформах на двухсторонних рынках с гетерогенными агентами и ограничениями на размер рынка / / Математическая Теория Игр и ее Приложения, - 2018. - Т. 10, - вып. 1. - c. 83-98.

232. Mazalov V., Chirkova J. Networking Games. Network Forming Games and Games on Networks. Academic Press, - 2019. - 322 p.

233. Petrosyan L.A., Mazalov V.V., Zenkevich N.A. (eds.). Frontiers of Dynamic Games: Game Theory and Management, St. Petersburg, 2018. Springer, -2019 . $-336 \mathrm{p}$. 
234. Mazalov V.V., Parilina E.M. Game of Competition for Opinion with Two Centers of Influence // 18th International Conference MOTOR 2019. LNCS, 2019. - Vol. 11548. - p. 673-684.

235. Mazalov V., Lukyanenko A. and Gurtov A. Location-Price Competition in Mobile Operator Market // International Game Theory Review, - 2019. Vol. 21, - no. 3 .

236. Seregina T.V., Ivashko A.A., Mazalov V.V. Optimal Stopping Strategies in the Game "The Price Is Right" // Proc. Steklov Inst. Math., - 2019. - Vol. 307. - p. 127-141.

237. Gusev V.V., Mazalov V.V. Potential functions for finding stable coalition structures // Operations Research Letters, - 2019. - Vol. 47. - p. 478-482.

238. Feng Z., Liu T., Mazalov V.V., Zheng J. Pricing of platforms in two-sided markets with heterogeneous agents and limited market size // Automation and Remote Control, - 2019. - Vol. 80, - issue 7. - p. 1347-1357.

239. Ford W., Lien J.W., Mazalov V.V., Zheng J. Riding to Wall Street: determinants of commute time using Citibike // International Journal of Logistics Research and Applications, - 2019. - Vol. 22, - no. 5. - p. 473-490.

240. Мазалов В.В., Дорофеева Ю.А., Коновальчикова Е.Н. Моделирование влияния среди участников образовательного коллектива // Вестник СПбГУ. Прикладная математика. Информатика, - 2019. - Т. 15, - вып. 2. - c. $259-273$.

241. Мазалов В.В., Хитрая В.А. Модифицированное значение Майерсона для определения центральности вершин графа // Математическая Теория Игр и ее Приложения, - 2019. - Т. 11, - вып. 2. - с. 19-39.

242. Мазалов В.В., Трухина Л.И. Письмо в редакцию // Дискретная математика, - 2019. - Т. 31, - вып. 2. - с. 159.

243. Серёгина Т.В., Ивашко А.А., Мазалов В.В. Стратегии оптимальной остановки в игре "The price is right"// Труды института математики и механики УрО РАН, - 2019. - Т. 25, - №3. - с. 217-231.

244. Мазалов В.В., Никитина Н.Н., Печников А.А. Сообщества в коммуникационном графе тематического фрагмента веба (на примере $\mathrm{PAH}$ ) /// XIII Всероссийское совещание по проблемам управления ВСПУ-2019, - 2019. - c. 2963-2967.

245. Kondratev A.Y., Mazalov V.V. Tournament solutions based on cooperative game theory // International Journal of Game Theory, - 2020. - Vol. 49(1). - p. 119-145.

246. Mazalov V.V., Ivashko A.A. Online Auction and Optimal Stopping Game with Imperfect Observation // Intelligent Information and Database Systems. ACIIDS 2020, LNCS, - 2020. - Vol. 12033. - p. 145-156.

247. Gao H., Mazalov V.V., Xue J. Optimal Parameters of Service in a Public Transportation Market with Pricing // Safety, Behavior, and Sustainability under the Mixed Traffic Flow Environment. Journal of Advanced Transportation, - 2020. - Vol. 2020.

248. Mazalov V.V., Parilina E.M. The Euler-Equation Approach in AverageOriented Opinion Dynamics // Mathematics, - 2020. - Vol. 8(3), - no. 355. - p. $1-16$. 